\title{
Nanocavities:
}

\section{Optomechanics goes molecular}

Mikołaj K. Schmidt and Javier Aizpurua

A theoretical framework that interprets Raman scattering as an optomechanical process can be used to understand, and guide, experiments in surface-enhanced Raman spectroscopy.

Surface-enhanced Raman spectroscopy (SERS) is a technique capable of detecting and chemically identifying single molecules in the vicinity of nanostructured metal surfaces. In a typical Raman process, a photon is inelastically scattered from a molecule, exchanging energy with its vibrations and carrying away its molecular fingerprint encoded in the loss or gain of energy. In SERS, this signal is thought to be enhanced due to localized plasmonic fields created by the nanostructured surface, which boosts the electric field of both the incident and scattered light; there is also thought to be a chemical contribution to the enhancement, which is connected with the adsorption of the molecules to the metallic interface. Over the past few years, the quality of SERS substrates has improved rapidly and has been accompanied by notable advances in measurement techniques. This has led to a number of experimental results that escape interpretation within standard theoretical formalisms. Writing inNature Nanotechnology, Christophe Galland, Tobias Kippenberg and colleagues at the École Polytechnique Fédérale de Lausanne now suggest that a framework derived from the seemingly unrelated domain of cavity optomechanics can be used to interpret SERS and shed light on these latest results.

In a typical optical cavity, formed by two highly reflective mirrors, circulating photons exert a radiation pressure on the mirrors. In optomechanical cavities, this force is harnessed by allowing one of the mirrors to move (Fig. 1a). The mechanical motion shifts the resonance frequency of the cavity, modifying the intensity of the circulating light and, therefore, the radiation pressure. This optomechanical 'backaction' can be considered as a feedback mechanism to quench or amplify the mirror vibrations (Fig. 1b). For example, if the cavity is illuminated by a laser that is red-detuned from its resonance, some of the vibrational energy will be transferred to the optical cavity and the mechanical vibrations will be quenched (or cooled). Alternatively, under a blue-detuned illumination, amplification (or heating) of the mirror vibrations will occur. The backaction mechanism has been successfully applied to cool the vibrations of mirrors in a variety of realizations of optomechanical cavities in the kilohertz to gigahertz frequency regime, where there is a large population of vibrations at thermal equilibrium. 
Figure 1: Cavity optomechanics in the classical and molecular regimes

a
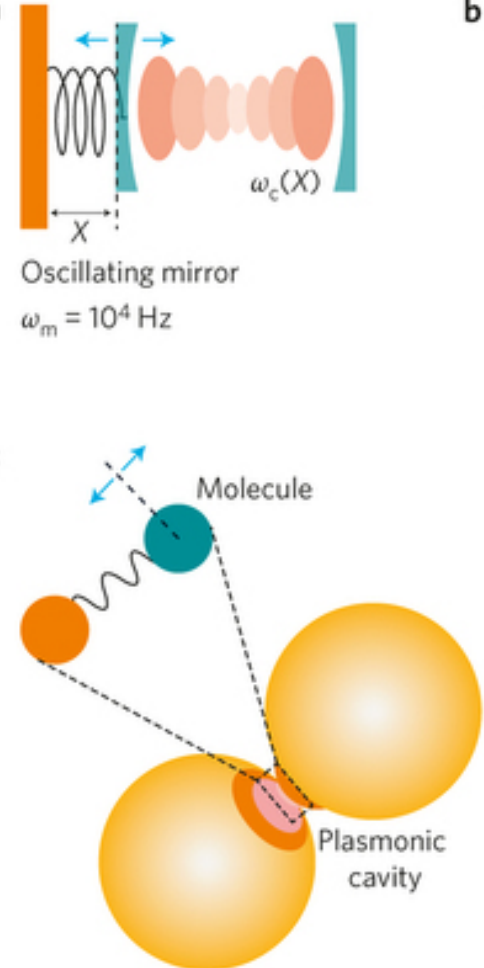

Plasmon-enhanced Raman scattering $\omega_{m}=10^{13} \mathrm{~Hz}$ b Cooling

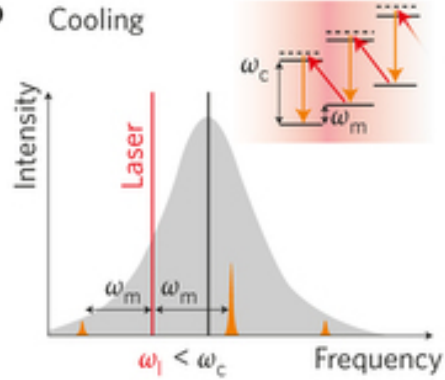

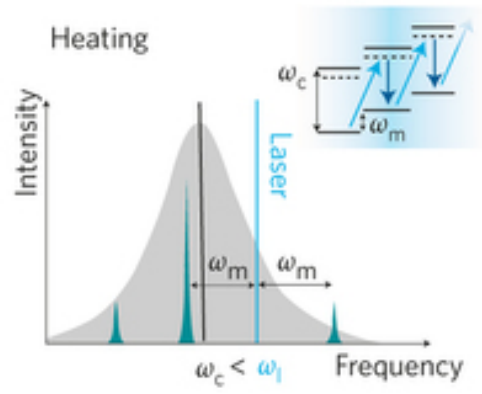
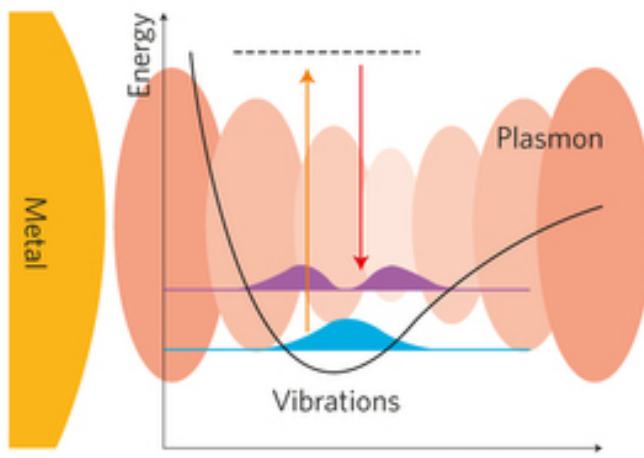

Coordinate $X$

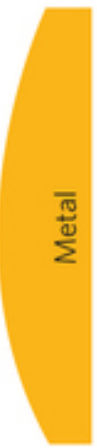

एल

a, Schematic of an optomechanical cavity with a resonant frequency $\omega_{\mathrm{c}}$ that depends on the displacement $X$ of a mirror. The mechanical frequency $\left(\omega_{\mathrm{m}}\right)$ of the mirror is shown. The finesse of the optical cavity is $10^{4}$. b. Sideband heating (right) or cooling (left) of the vibrations is achieved by illuminating the cavity with a laser of frequency $\omega_{1}$, blue- or red-tuned, respectively, from the resonance of the empty cavity $\left(\omega_{c}\right)$. The corresponding insets show a simplified level structure of both processes, where the dashed lines denote virtual states. c, Plasmon-enhanced Raman process in the context of optomechanics. Left: A plasmonic cavity with quality factor $Q=$ 10 acts as a 'bad' optical cavity and the molecular vibrations of a molecule located in it act as a high-frequency mechanical oscillator. Right: In the off-resonant Raman scattering, the transition between two vibrational states (blue and purple waveforms) of the ground electronic state of the molecule (solid black line as a function of coordinate $X$ ) is driven by the cavity plasmons (orange and red arrows). When the frequency of the plasmonic modes is significantly smaller than the energy gap of the molecular electronic states, the transition is mediated by a virtual state (dashed line).

Kippenberg and colleagues suggest that a direct analogy can be found between the optomechanical backaction taking place in optical cavities (Fig. 1a) and the interaction of molecular vibrations and localized plasmons found in SERS (Fig. 1c). The two can be connected by considering an inelastic off-resonant Raman process as an optomechanical system in which molecular vibrations modify the energy of the plasmonic cavity. 
This insight allows the coherent nonlinear interaction between quantized plasmons and molecular vibrations in SERS to be described using a typical optomechanical Hamiltonian that couples the population of the cavity with the vibrational degree of freedom.

The strength of this coupling is determined by the Raman activity of the molecule and the effective mode volume into which the plasmonic cavity can squeeze light. As a result, a SERS system can be considered as an optomechanical set-up with very high mechanical frequencies of the vibrations (tens of terahertz) and significant coupling parameters, in spite of the bad quality factors of plasmonic cavities.

This model suggests that the vibrations of a molecule can be amplified in SERS by illuminating a plasmonic cavity with a blue-detuned laser, just like the heating of mirror vibrations in an optomechanical cavity can be induced by a similarly detuned laser. Under these conditions, a significant increase of the Raman signal intensity is expected. In fact, Kippenberg and colleagues point out that such an effect may have been observed in recent Raman experiments in which blue detuning of the incident laser light was required for maximum enhancement ${ }^{2}$, and an increase in power intensity led to a narrowing of the spectral signal ${ }^{3}$. While these results would not be predicted by standard SERS theory, they can be foreseen and explained by this new model. This theoretical framework also has implications for future SERS experiments. In particular, the relative position of a vibrational band with respect to the energy of the plasmonic cavities and the incident laser should be taken into consideration.

Furthermore, special attention should be paid to the analysis of nonlinear signals involving stimulated emission of vibrations ${ }^{\underline{5}}$, as well as the correlations between the Stokes (which occurs when a molecule gains energy) and anti-Stokes (which occurs when a molecule loses energy) photon scattering. The low thermal populations of vibrations and large coupling parameters could enable the control of entanglement between the states of the optical cavity and vibrations, as well as the study of fundamental optomechanical effects related to quantum correlations and metrology. Molecular spectroscopy suddenly opens the door to explore the interactions of light and vibrational states of matter at the quantum level, thus bringing optomechanics to the nanoscale. 